\title{
Unmanned Aerial Vehicle Based Wireless Sensor Network for Marine-Coastal Environment Monitoring
}

\author{
Carlos Andrés Trasviña Morenoํㅜ Rubén Blasco Marín ${ }^{1}$, Álvaro Marco Marco ${ }^{1}$, \\ Roberto Casas Nebra ${ }^{1}$ \\ ${ }^{1}$ Human Openware Research Lab (HOWLab) \\ Instituto de Investigación en Ingeniería de Aragón (I3A) \\ Universidad de Zaragoza, Mariano Esquillor s/n, 50018, Zaragoza, Spain. \\ Tel. +34-876555464, e-mail: ctrasvina@ humanopenware.com
}

\begin{abstract}
A UAV-bouy hybried Wireless Sensor Network, with LoRa communications, is presented for the monitorization of marine-coastal environments. This implementation offers a low-power, long-range and cost-effective solution to current marine monitoring applications, as well as a safer method to observing natural protected areas and reservoirs.
\end{abstract}

\section{Introduction}

Marine environments are one of the harshest settings to monitor for any type of deployment, as it requires waterproof robust technology to endure the high levels of humidity and salinity, wave collisions, and extreme weather conditions. Buoy implementations are the most commonly used, being applied to ambient variable measurement. These devices can measure several variables simultaneously and monitor areas for long periods of time. One of their main drawbacks is the process for data extraction. The most commons methods are long-range communications, close-range wireless transmissions, or dry-land data collection. Long-range (LoRa) transmissions require either a previously established infrastructure, such as a ground base station, or satellite connectivity [1].

One of the most novel approaches for monitoring marine environments is the use of Unmanned Aerial Vehicles (UAV). As they are airborne devices, UAVs do not face the same challenges as marine monitoring deployments [2]. Implementations using these types of devices have been used for wildlife, environmental, and hazardous marine monitoring. Yet, given their short life span of tens of minutes and the need for user interactions, monitoring for extended periods of time is difficult to achieve, as would requires several landings and battery replacements.

To overcome the limitations of UAV and buoy based implementations, the use of a hybrid Wireless Sensor Network (WSN), which uses both technologies, is proposed in this article. The system proposed in this article aims to use a swarm of buoys as primary environmental sensors, covering wide areas of seashore, and a UAV as a data sink and dynamic network router. To communicate the buoys with the UAV, the LoRa spread spectrum modulation [3], a Low Power Wide Area Network (LPWAN) protocol, was selected. This type of communication offers highly configurable long range communications with low power consumption, and a high resilience to external interference.

\section{Functional Overview}

Two main functional scenarios can be described for this implementation: data extraction and search and rescue. In both cases, the buoys will periodically collect environmental data and GPS coordiantes. In the data extraction scenario, after the buoys have been set on the shore line and ambient variables have been collected, the UAV will fly in the last known direction of the buoys, periodically searching for them. Once the buoys have been found, the UAV will automatically start transmitting data acquisition messages to collect the data stored in the buoys, and finally return to home.

In the search and rescue scenario, the UAV will be used to locate missing buoys that may have drifted away from the rest of the swarm. Given that the buoys may be either stranded or in a distant location, the UAV will be flown in the suspected direction of the buoys and will try to collect the position of any buoy in its path before its return to home. This functionality simplifies the recovery of the buoys, 
which, in many cases, are lost to strong currents or vandalism [1].

\section{System Architecture}

Two distinct hardware were developed with LoRa wireless communications: the control node and the sensor node. The control node functions either as a network master, mounted on the UAV, or as a network monitor in a ground station; the sensor node hardware can only be placed inside the buoy. For both hardware, a firmware was developed with a custom network layer protocol which will be referred to as LoRaNET. Additionally, to be able to interact with the SIMMA system, a Graphical User Interface (GUI) was also developed to simplify the process of configuration. The buoys are based on a design of the Davis drifter buoy, which has an "X" style sail and four floatation devices. The UAV is based on a Delta Wing design, built with a foam frame with two carbon fiber rods across the wings for reinforcement and with a wingspan of $2 \mathrm{~m}$.

\section{Tests and Results}

To validate the system, a prior experimentation was done before the field test validation, as well as several tests for each of the components of the system. In a controlled environment, the wireless communications were tested in conjunction with the UAV, as it was critical to verify possible interferences to the UAV. A buoy was placed in dryland and the UAV was flown in a circular pattern, in both manual and automatic modes, requesting data to the buoy.

After the succesfull results from prior tests, a final field test was conducted in the beach of El Tecolote (La Paz, B.C.S., México), where three buoys where placed near the shoreline collecting environmental data. Afterwards, the UAV was launched from the beach and automatically flown in a pre-defined flight pattern, searching for buoys and extracting the sensor data (Figure 1). With LoRa settings used in this test, one that has a low transmission range, the UAV could successfully communicate with the buoys at approximately $4 \mathrm{~km}$ in distance. Although greater transmission ranges are achievable $(20+\mathrm{km})$, this trial allowed for a safe validation of the system as a whole, in a relatively controlled environment.

\section{Conclusions}

In this article, the design and implementation of a UAV assisted long-range buoy based WSN for the monitorization of marine-coastal environments, as well as an improvement to current implementations, has been proven. This development provides an easyto-use and low power solution, with an extended range of several kilometers, as well as a more costeffective alternative with fewer infrastructure requirements. Furthermore, it serves as a less invasive method for monitoring natural protected areas and reservoirs.

The integration of the UAV, the Davis monitoring buoys, and the LoRa communications as a single system, adds a needed flexibility to current implementations and the possibility of studying previously unreachable areas, since the buoys can be placed with a small vessel and the UAV can be flown from almost any area, given its great communications reach.

\section{REFERENCES}

[1]. XU, G., SHEN, W., AND WANG, X. Applications of wireless sensor networks in marine environment monitoring: A survey. Sensors. 2014, 14, pp. $16932-$ 16954.

[2]. HODGSON, A., KELLY, N., PEEL, D. Unmmanned aerial vehicles (UAVs) for surveying marine fauna: A dugong case study. PLoS ONE. 2013, 8, e79556.

[3]. AUGUSTIN, A., YI, J., CLAUSEN, T., TOWNSLEY, W. A study of LoRa: Long range \& low power networks for internet of things. Sensors. 2016, 16, 1466.

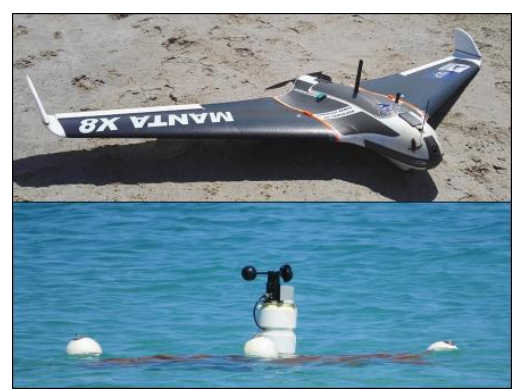

Figure 1 - UAV and buoys used in the field test 